\title{
La ricerca in psicoterapia: il contributo del Gruppo di lavoro della Cattedra di Psicologia Dinamica (base) della Seconda Università di Napoli
}

\author{
Giorgio Caviglia, ${ }^{1}$ Raffaella Perrella, ${ }^{2}$ Walter Sapuppo, ${ }^{3}$ \\ Nadia Del Villano 4
}

\section{Sommario}

Lo scopo del presente lavoro è quello di illustrare i contributi passati e gli attuali interessi del Gruppo di ricerca della Cattedra di "Psicologia Dinamica" (corso di base) della Facoltà di Psicologia della Seconda Università di Napoli, nonché di presentare e argomentare le pubblicazioni scaturite negli ultimi anni dalle quelle ricerche.

I lavori si inseriscono nelle aree relative alla Teoria dell' Attaccamento, alla valutazione psicologica, allo studio della psicopatologia connessa ai Disturbi del Comportamento Alimentare, alla ricerca in psicoterapia "single case".

\section{Parole chiave}

Disturbi del comportamento alimentare, teoria dell'attaccamento, ricerca in psicoterapia, psicopatologia

\footnotetext{
${ }^{1}$ Professore Associato, Dipartimento di Psicologia, Facoltà di Psicologia, S.U.N.,

2 Assegnista di Ricerca, Professore a contratto, Facoltà di Scienze della Formazione, Università degli Studi di Urbino "Carlo Bo", 3Docente contrattista, Facoltà di Psicologia, S.U.N., Scuola di Psicoterapia Cognitiva SPC-Napoli, ${ }^{4}$ Dottorando di Ricerca, Dipartimento di Scienze Pedagogiche, Università degli Studi di Bari
}

Corrispondenza: Prof. Giorgio Caviglia, Dipartimento di Psicologia, Facoltà di Psicologia, S.U.N., via Vivaldi 43 -81100- Caserta (CE)

E-mail: giorgio.caviglia@unina2.it 


\section{Introduzione}

La Cattedra di "Psicologia Dinamica" (corso di base) della Facoltà di Psicologia della Seconda Università di Napoli si occupa da diversi anni di ricerca in psicoterapia e in particolare della ricaduta teorica (rispetto ai costrutti e ai modelli) e clinica (rispetto alle tecniche collegate a una teoria) dei risultati della ricerca empirica. Il Referente e Coordinatore è il Prof. Giorgio Caviglia, Professore Associato confermato di "Psicologia Dinamica". Collaboratori a ricerche specifiche sono stati, negli anni, Raffaella Perrella, Walter Sapuppo, Nadia Del Villano, Sara Bisogno, Claudia Cecere, Domenico Nardiello, Marco La Marra, Emanuele Del Castello, Barbara Fiocco, Adriana Solla. I collaboratori sono tutti Psicologi che hanno diverse qualifiche (Professori a contratto, dirigente psicologo della ASL, Dottori di Ricerca e dottorandi, consulenti di Tribunale, di aziende e di studi privati) e specializzazioni (in Psicoterapia Psicoanalitica, Psicodinamica, Lacaniana, Relazionale, Cognitivo-Comportamentale e in Psicodiagnostica). La Sede del Gruppo di ricerca è presso la Seconda Università degli Studi di Napoli, Facoltà di Psicologia, Dipartimento di Psicologia.

La ricerche portate avanti dalla Cattedra, hanno sempre avuto come obiettivo quello di proporre una loro successiva ricaduta sia sulle teorie a cui si riferivano e da cui derivavano, sia a livello dell'intervento clinico.

Nella bibliografia finale verranno riportati tutti i lavori, relativi alle diverse aree, sviluppati nel tempo dagli appartenenti al Gruppo di ricerca. I lavori prodotti verranno divisi, per chiarezza espositiva, come detto, in cinque filoni. Abbiamo deciso di raggruppare gli ambiti di ricerca del nostro Gruppo di lavoro nelle seguenti cinque aree principali:

1) Disturbi del Comportamento Alimentare (la tematica più recente);

2) teoria dell'Attaccamento applicata all'ambito scolastico e clinico;

3) nonché come riflessione storica, teorica e clinica;

4) ricerca in psicoterapia "single case";

5) trauma e psicopatologia; 
6) ambito psicodiagnostico e della valutazione psicologica.

Vediamo, a seguire, in maniera più dettagliata, le aree specifiche, le cui citazioni sono inserite, come già detto, tutte insieme, nella bibliografia finale.

\section{1) Disturbi del Comportamento Alimentare e obesità}

Per quanto concerne i Disturbi del Comportamento Alimentare (DCA), attualmente il Gruppo è impegnato sia nella ricerca, all'interno di campioni clinici, di relazioni tra tali disturbi, fenomeni dissociativi e difficoltà relative all'espressione delle emozioni (alessitimia) (Caviglia, 2009), che nella valutazione dell'obesità come "disturbo mentale" e/o comportamentale.

Inizialmente il Gruppo era indirizzato a campioni particolari, che abbiamo ritenuto avrebbero potuto essere "a rischio" di DCA, per l'estrema attenzione e valore dati all'immagine corporea (Caviglia, Perrella, Bisogno, \& La Marra, 2006; La Marra, Bisogno, Perrella, \& Caviglia, 2006; La Marra, Perrella, Bisogno, \& Caviglia, 2006), quali gli operatori dello spettacolo.

E' stato successivamente studiato un campione di adolescenti campani (Caviglia, Perrella, La Marra, \& Giannini 2006), rispetto ai fenomeni e alle difficoltà summenzionate, ottenendo risultati positivi che non disconfermano i dati riportati della letteratura internazionale; in seguito, in uno studio pubblicato sul Volume 12 (Numero 1/2, Novembre-Dicembre 2009) di "Ricerca in Psicoterapia" (La Marra, Sapuppo, \& Caviglia, 2009b), sono state valutate le relazioni che intercorrono tra i risultati ottenuti all' Eating Disorder Inventory-2 (EDI2; Garner, 1991), alla Dissociative Experiences Scale (DES; Bernstein \& Putnam, 1986) e alla Scala Alessitimica Romana (SAR; Baiocco, Giannini, \& Laghi, 2005) in un campione di 53 pazienti adulti con diagnosi di Disturbo del Comportamento Alimentare (14 con diagnosi di Anoressia Nervosa, 15 con Bulimia Nervosa, 12 con DCA Non Altrimenti 
Ricerca in Psicoterapia / Research in Psychotherapy 2010; 2 (13): 32-52 http://www.researchinpsychotherapy.net

Specificato e 12 con Disturbo da Alimentazione Incontrollata). I dati emersi dalla nostra ricerca, ancora una volta, non disconfermano le relazioni riportate in letteratura tra $\mathrm{i}$ Disturbi del Comportamento Alimentare, i fenomeni dissociativi e l'alessitimia (cfr. anche: Caviglia, Bisogno, Perrella, \& La Marra, 2006; La Marra, Piombino, Messina, Perrella, Chieffi, Mangoni di Santo Stefano, \& Caviglia, 2006).

Attualmente il Gruppo è impegnato, oltre che nell'ampliamento del campione, anche nel reclutamento di soggetti gravemente obesi per valutare eventuali caratteristiche discriminanti. A tal proposito, dopo un'attenta disamina dei maggiori contributi teorici riguardo al significato e all'etiopatogenesi dell'obesità, sono stati condotti altri studi volti a comprendere gli aspetti psicopatologici comunemente associati ai DCA (dissociazione, immagine corporea, abitudini alimentari e alessitimia) di un gruppo di 561 soggetti adolescenti campani rispettivamente ripartiti nelle diverse categorie (normopeso, sovrappeso e obesi) definite dall' Indice di Massa Corporea (IMC o BMI, Body Mass Index) e senza diagnosi di DCA. Dai risultati emersi dal nostro studio (La Marra, Sapuppo, \& Caviglia, 2009a) si evince come alcune delle dimensioni psicologiche comunemente associate ai DCA, quali l'impulso alla magrezza, la mancanza di consapevolezza enterocettiva, l'insoddisfazione per il corpo, la mancanza di empatia, l'insicurezza sociale, le eccessive preoccupazioni per il proprio aspetto fisico, il controllo compulsivo dell'aspetto fisico, l'inadeguatezza, la sfiducia interpersonale e l'insoddisfazione per le parti corporee che vedono coinvolte la testa e il tronco, siano in grado di spiegare una porzione consistente della varianza del BMI di un gruppo di soggetti ai quali non è stato diagnosticato alcun disturbo alimentare secondo i criteri diagnostici del DSM IV-TR. A queste, seppur in misura minore, si associano l'autodirezionalità, la tendenza ad un'alimentazione non corretta e l'incapacità di gestire il comportamento alimentare. Si escludono dall'equazione, quindi, perché il criterio statistico adottato 
non evidenzia una probabilità associata statisticamente significativa, i tratti temperamentali indagati secondo il modello di Cloninger, la presenza di esperienze dissociative e le dimensioni chiave che definiscono il costrutto dell'Alessitimia e ampiamente documentate in letteratura come profondamente associate ai DCA (cfr. anche: La Marra, Sapuppo, Messina, Mangoni di S. Stefano, Chieffi, \& Caviglia, 2009; La Marra, Sapuppo, \& Caviglia, 2010; La Marra, Sapuppo, Chieffi, \& Caviglia, 2010).

Tutti gli studi vanno inquadrati all'interno di una cornice teorico/clinica che vede la lettura prospettica multidimensionale dei DCA come il miglior approccio, sia dal punto di vista della spiegazione eziopatogenetica, che del mantenimento del disturbo, che dell'intervento terapeutico (cfr. Caviglia \& Cecere, 2007).

\section{2) Teoria dell' Attaccamento}

a) Contributi riguardo lo sviluppo sociale, emotivo e cognitivo Per quanto concerne l'attaccamento, il nostro Gruppo è stato ed è impegnato su diversi fronti di ricerca, tutti derivanti da continuativi interessi e riflessioni teoriche (Brisch, 2005; Caviglia, 2003; Caviglia, 2005; Caviglia \& Bisogno, 2008; Del Villano \& Bisogno, 2009).

Particolare attenzione è stata rivolta al ruolo che l'attaccamento madrebambino e quello educatrice-bambino giocano nello sviluppo dei bambini e, allo stesso tempo, alle variabili che influenzano la qualità di tale legame (Cassibba \& Caviglia, 2000; Caviglia, Cassibba, \& Coppola, 2000). Dai nostri studi si evidenzia, inoltre, che le madri classificate come "sicure" (attraverso l'utilizzo dell' Adult Attachment Interview; George, Kaplan, \& Main, 1985) hanno bambini che manifestano maggiori competenze socio-emotive (valutate con la Socio-Emotional Dimension Scale; SEDS, Hutton \& Roberts, 1986), se paragonati a bambini figli di madri "insicure" (Caviglia, Solla, \& Dazzi, 2003). Inoltre, sia la sensibilità dell'educatrice, che la qualità delle strutture 
istituzionali (scuole elementari e asili nido), sembrano costituire un terreno ideale per lo sviluppo di un attaccamento sicuro del bambino (Caviglia \& Pili, 2001). Per valutare i costrutti implicati, sono stati utilizzati diversi strumenti:

- il Maternal Behaviour Q-set (Pederson \& Moran, 1995), un test composto da 90 item ciascuno dei quali descrive un comportamento specifico dell'educatrice in risposta a segnali specifici del bambino;

- l'Attachment Q-set (Waters \& Deane, 1985), un test composto da 90 item che descrive un comportamento specifico di attaccamento del bambino nei confronti dell'educatrice;

- la SVANI (Scala per la Valutazione dell'asilo nido; Harms, Cryer, \& Clifford, 1990), una scala formata da 37 item che fornisce un quadro complessivo della qualità del servizio offerto della struttura presa in considerazione.

Negli ultimi 3 anni, l'attività di ricerca della Cattedra si è focalizzata sulle relazioni tra Memoria di Lavoro (Working Memory, WM), stile di attaccamento e apprendimento/rendimento scolastico. Come ampiamente affermato in letteratura, il processo di apprendimento coinvolge sia numerose abilità cognitive - quali attenzione, memoria, linguaggio - che competenze relazionali-sociali. Numerose ricerche evidenziano una marcata associazione tra deficit della Memoria di Lavoro (ML) e disturbi dell'apprendimento nei bambini. Numerosi studi rilevano che le abilità della ML influiscono, in particolar modo, sull'apprendimento del linguaggio scritto e dell'aritmetica.

Sulla base degli studi effettuati nell'ambito della Teoria dell'Attaccamento è possibile evidenziare che bambini con un attaccamento sicuro con il proprio caregiver e/o con il/la proprio/a insegnante, dimostrano migliori capacità nell'espressione e riconoscimento emotivo, nel comportamento prosociale, nelle attività ludico-cognitive, nell'acquisizione di concetti base, nell'adattamento scolastico e nello sviluppo linguistico-espressivo. 
Inizialmente è stata valutata empiricamente la presenza di relazioni significative tra $\mathrm{i}$ risultati ottenuti in una serie di prove cognitive quantificanti la ML - e il rendimento scolastico, rispecchianti la qualità del livello di apprendimento raggiunto (Caviglia, La Marra, Sapuppo, \& Perrella, 2010).

Inoltre, per sottoporre ad analisi correlazionale i risultati ottenuti al test con il rendimento scolastico, sono state rilevate le valutazioni degli insegnanti rispetto agli insegnamenti di Italiano e Matematica di 89 dei nostri 100 soggetti reclutati. Gli 11 soggetti esclusi non rispettano i criteri di inclusione dello studio, a causa della frequentazione della scuola dell'infanzia dove non è presente, come nella scuola primaria, la valutazione numerica decimale (Legge 169/2008). Tuttavia i soggetti più piccoli sono stati inclusi nello studio per l'adattamento italiano.

A oggi (La Marra, Perrella, Marciano, Cecere, Intoccia, Ciccarelli, Del Villano, Bisogno, \& Caviglia, 2009; Del Villano, Sapuppo, Cecere, La Marra, Perrella, Marciano, Intoccia, \& Caviglia, 2010), ai fini di una più approfondita indagine, sono stati somministrati i seguenti test:

- Test di Valutazione Linguistica (TVL) (Cianchetti \& Sannio Fancello, 2007), allo scopo di quantificare il livello evolutivo complessivo del bambino, nonché di evidenziare eventuali differenze e/o anomalie nell'evoluzione delle varie componenti ed espressioni linguistiche;

- Separation Anxiety Test (SAT) nella versione "famiglia" (Attili, 2001) e "scuola" (Liverta Sempio, Marchetti, \& Leccio, 2001). Un test semiproiettivo basato su una serie di figure che rappresentano scene di separazione tra un bambino/bambina e i suoi genitori e tra un bambino/bambina e la sua insegnante. Si ipotizza che queste scene attivino le rappresentazioni del bambino legate all'attaccamento, permettendo di classificarle.

Dall'elaborazione dei dati raccolti attraverso la somministrazione del TVL è emerso, in linea generale, un corretto sviluppo linguistico e l'assenza di significativi deficit linguistici. I punteggi ponderati emersi 
Ricerca in Psicoterapia / Research in Psychotherapy 2010; 2 (13): 32-52 http://www.researchinpsychotherapy.net

dal nostro campione sono compresi da 3.5 a 9.4 (M 7.3) e DS 0.20. Attualmente gli autori sono impegnati nell'elaborazione dei dati relativi alla valutazione dei Modelli Operativi Interni attraverso il SAT.

b) Contributi riguardo tematiche generali e cliniche

L'interesse per la Teoria dell'Attaccamento si è esplicato sia a livello di riflessione teorica, che di ricaduta clinica (Brisch, 2005; Caviglia, 2003; Caviglia, 2005; Caviglia \& Bisogno, 2008). Per rendere operativa l'applicazione della Teoria a livello di ricerca, tre membri del Gruppo hanno partecipato ai Corsi dell" "Official Adult Attachment Training Institute", che si sono tenuti a Roma, in diversi anni e hanno superato $\mathrm{i}$ "reliability testing" previsti (30 casi in Inglese), con esito "full pass" (categorie F, Ds, E, U) e giudizio "highly reliable". L'Attaccamento è stato dunque utilizzato come chiave di lettura teorica, nello sviluppo normale e patologico, e come strumento di ricerca.

In un primo momento, particolare attenzione è stata rivolta alle pazienti agorafobiche rispetto alle quali è stato evidenziato come lo "stato della mente" rilevato attraverso l'AAI sia statisticamente identificabile come un attaccamento di tipo "insicuro" (Caviglia, Del Castello, \& Curcio, 2001; Caviglia, Del Castello, \& Fiocco, 2001; Caviglia, Del Castello, \& Fiocco, 2002), dato che non disconferma l'intuizione di Bowlby (1973). Inoltre è emerso, altro dato interessante della nostra ricerca, che anche i partner delle donne agorafobiche risultavano "insicuri" all' AAI, in maniera statisticamente significativa, dato che non disconferma l'ipotesi di Liotti e Guidano (1976).

Successivamente, l'interesse si è spostato sulla correlazione fra traumi infantili, attaccamento adulto e Attività Referenziale, misurata con lo strumento DAAP (Dizionario Ponderato dell'Attività Referenziale) (Tagini, Pazzagli, Caviglia, \& Dazzi, 2007; Tagini, Pazzagli, Caviglia, Pravato, De Coro, \& Dazzi, 2009). I risultati della ricerca, condotta su 4 soggetti (appartenenti a un campione più ampio di 65 studenti) i cui 
trascritti erano stati siglati con la AAI, il DAAP e il Childhood Trauma Questionnaire (CTQ), hanno mostrato numerose e significative attivazione sub-simboliche dell'AR, in concomitanza di eventi narrativi in cui venivano riportati i maltrattamenti e i traumi subiti.

Altro significativo campo di ricerca clinico con l'Attaccamento, è stata l'applicazione dell'AAI ai "Figli della Shoà" (Caviglia, Gangi, \& Fiocco, 2002; Caviglia \& Fiocco, 2003; Caviglia, Fiocco \& Fullone, 2003; Caviglia, Fiocco, Solla \& Dazzi, 2004; Caviglia, Fiocco, \& Dazzi, 2004; Caviglia \& Bisogno, 2009), cioè la seconda generazione dei figli dei sopravvissuti ai campi di sterminio nazisti. Lo scopo della ricerca e dei lavori scaturiti era quello di indagare quale contributo la prospettiva dell'attaccamento poteva portare alla comprensione della trasmissione intergenerazionale del trauma della Shoà. In particolare, l'ipotesi era di studiare come e se la dissociazione, l'absorbtion, i comportamenti spaventati/spaventanti (Lyons-Ruth \& Atwood, 1997) dei Genitori vittime dell'Olocausto, potessero rappresentare l'equivalente operazionalizzato dell' “ombra dell'Olocausto" (Moses, 1993) sui Figli. Il campione era costituito da 26 ebrei italiani, i cui genitori erano tornati dai campi di sterminio e 26 ebrei italiani, comparabili per età, sesso e livello socio-economico-culturale, i cui genitori erano sopravvissuti allo sterminio nazista, senza essere stati rinchiusi nei campi. Non sono risultate differenze significative fra il gruppo sperimentale e il gruppo di controllo. Ma sebbene il gruppo sperimentale non fosse significativamente "traumatizzato" (elemento inatteso), entrambi i gruppi mostravano una distribuzione delle categorie dell'AAI, diverse dalla distribuzione "normale" (van IJzendoorn \& BakermansKranenburg, 1996); inoltre, il gruppo sperimentale differiva solamente un po', per la frequenza della categoria "Dismissing", forse dovuta all'atteggiamento ritirato, duro e autosufficiente del Genitore, magari inconsciamente attuato anche per difendere il Figlio dal proprio trauma (che, infatti, non viene "automaticamente" trasmesso). 
Infine, l'ultimo campo di interesse di questo ambito di ricerca (l'AAI) ha come obiettivo di costruire un ponte, ponendo a confronto tre metodi diversi di "raccontare la propria vita", per attivare una riflessione comune, fra tre differenti campi d'indagine, quali la ricerca psicologicoclinica, la psicoterapia e la ricerca pedagogico-didattico (Caviglia, Del Villano, \& Sapuppo, 2010). Tale obiettivo vuole essere conseguito individuando ed evidenziando $\mathrm{i}$ punti di contatto e accostando tre metodologie di applicazione empirica, quali il Metodo NarrativoAutobiografico con adulti, l'Adult Attachment Interview (AAI) e la procedura di indagine psicoterapeutico-cognitiva denominata ABC. Scopo di questo lavoro è anche quello di evidenziare l'importanza della "narrazione di Sé", ai fini di un eventuale cambiamento (sia all'interno di un contesto psicoterapeutico, che quotidiano - lavorativo, familiare, etc.). Attraverso le narrazioni, infatti, sia nell'intervista sull'Attaccamento, che nel racconto autobiografico, che nei protocolli $\mathrm{ABC}$, il soggetto può collocare le sue azioni e i suoi pensieri in uno specifico tempo e in uno specifico spazio, e inscrivere se stesso in un rapporto di causa ed effetto o reciprocità con altre azioni, altri eventi ed altre persone, dando modo al comportamento passato di assumere un senso e un significato nuovi. In questo modo, operando una riflessione "meta", il soggetto può giungere a una maggiore conoscenza di se stesso e dei suoi modi di essere e di sentire.

\section{3) Ricerca in psicoterapia}

Relativamente all'area della "Ricerca in Psicoterapia" il Gruppo, focalizzando il proprio interesse sulle ricerche single-case (Caviglia, Scafuto, Solla, \& Semerari, 2003; Perrella, D'Amore, La Marra, \& Caviglia, 2005), si è occupato - e si occupa - della valutazione del processo e della valutazione dell'esito (note in letteratura come process research e outcome research) attraverso lo studio di percorsi e/o interventi psicoterapeutici realizzati con pazienti con specifiche 
patologie o deficit (Andreassi, Cascioli, Caviglia, De Coro, Lingiardi, Ortu, Pazzagli, Piscicelli, Williams, \& Dazzi, 2000; Caviglia, Scafuto, Perrella, \& Semerari, 2006).

Si suole, infatti, distinguere la ricerca in psicoterapia in due differenti settori: l'outcome research (ricerca sul risultato) - che fa riferimento alla ricerca sugli esiti del trattamento, misurabile al termine della terapia stessa (in termini di differenze tra lo stato pre e post-terapia valutate con determinati strumenti standardizzati) - e il process research (ricerca sul processo) che riguarda, invece, la ricerca sui vari aspetti del "processo" della terapia, i quali possono essere misurati anche mentre essa è in corso e indipendentemente dal risultato. In questo ambito di ricerca si inserisce l'Attività Referenziale (AR) (Bucci, 1997; Caviglia \& De Coro, 2000; De Coro, Ortu, Caviglia, Andreassi, Pazzagli, Mariani, Visconti, Bonfanti, Bucci, \& Maskit, 2004), che si può definire come l'attività del sistema delle connessioni referenziali tra sub-simbolico, rappresentazioni simboliche verbali e non verbali, e come queste connessioni si riflettono nello stile del linguaggio. Le misure dell'Attività Referenziale valutano il grado in cui chi parla, o chi scrive, è capace di tradurre tali esperienze in parole, così da evocare esperienze corrispondenti nell'ascoltatore o nel lettore. Il livello di $A R$ presenta una variabilità sia di stato che di tratto, indica cioè, cambiamenti in uno stesso soggetto nel corso del tempo, in funzione di eventi esterni o interni, o differenze interindividuali più stabili (De Coro \& Caviglia, 2000; Caviglia, 2001). Date queste premesse, in questo contesto teorico la psicopatologia può essere ricondotta alla disconnessione tra i sistemi di elaborazione delle informazioni e alla dissociazione degli schemi emotivi (Caviglia \& Del Villano, 2010). Tali variazioni sono state rilevate all'interno della terapia mettendo a punto un metodo empirico per la valutazione dell'Attività Referenziale. L'AR può essere misurata attraverso il metodo manuale applicato da giudici che hanno seguito un 
training di addestramento, o attraverso $i$ più recenti metodi computerizzati (cfr. nel §2b il DAAP).

Nel campo della ricerca in psicoterapia, il Gruppo si è focalizzato anche sullo studio del costrutto della metacognizione operazionalizzato e valutato attraverso la Scala di Valutazione della Metacognizione (S.Va.M.) (Carcione, Falcone, Magnolfi, \& Manaresi, 1997) - che risulta essere un utile ponte di raccordo fra le diverse concettualizzazioni che cercano di spiegare l'eziopatogenesi del Disturbo Borderline di Personalità (DBP) (Caviglia, Iuliano, \& Perrella, 2005).

Partendo dallo studio di diversi approcci teorici che mirano alla comprensione della genesi del disturbo borderline (Caviglia, Iuliano, \& Perrella, 2005), e all' individuazione dei conseguenti interventi psicoterapeutici possibili, attraverso il costrutto della meta cognizione, ci siamo posti (Caviglia, Solla, Scafuto, \& Semerari, 2003; Caviglia, Scafuto, Perrella, \& Semerari, 2006) l'obiettivo di analizzare se esista una tipologia di deficit metacognitivo specifica del disturbo borderline di personalità e se si rilevi un incremento metacognitivo nel corso di una psicoterapia, tale da essere indicativo del grado d'efficacia della psicoterapia stessa.

Dall'analisi delle frequenze rilevate, abbiamo osservato- attraverso uno studio sul processo psicoterapeutico di un single-case - un miglioramento delle funzioni metacognitive progressivo e globale, che non disconferma l'ipotesi di partenza. Nello specifico accade che la caratterizzazione, ossia la capacità di discriminare tra loro le componenti cognitive ed emotive dei propri stati interni, è positiva e costante in tutte le sedute esaminate; la reversibilità del pensiero ha valori deficitari nelle sedute iniziali e assume valori crescenti in quelle mediane, fino a raggiungere il picco positivo nelle ultime sedute; il riconoscimento dei limiti del pensiero segue invece un andamento crescente lineare; la relazione tra variabili, funzione del monitoraggio meta cognitivo, ha sempre valori positivi, tuttavia anch'essa migliora 
nelle sedute centrali, per poi restare costante nelle ultime; le funzioni dell'integrazione, da che erano molto basse, seguono lo stesso incremento positivo nel corso dello svolgersi della psicoterapia.

\section{4)Trauma e psicopatologia}

Il Coordinatore del Gruppo di ricerca si è da tempo concentrato sulla letteratura internazionale, che evidenzia prove empiriche sempre più numerose che confermano come esperienze traumatiche di abuso o maltrattamento infantile siano frequentemente associate a patologie accomunate da deficit nella modulazione delle emozioni e nell'integrazione del Sé (Caviglia, 1998; Tagini, Pazzagli, Caviglia, De Coro, \& Dazzi, 2008). La sofferenza emotiva indotta da avvenimenti traumatici non è, però, necessariamente legata alla dissociazione nei bambini, se questi avvenimenti si verificano all'esterno del contesto delle relazioni familiari. Il conflitto si manifesta, attraverso un comportamento disorganizzato, quando il bambino, subendo abusi e maltrattamenti dalle figure genitoriali, adotta strategie incoerenti e disfunzionali, espressione di rappresentazioni contraddittorie e non integrate del Sé e della figura di attaccamento. Gli studi longitudinali esposti dimostrano come la teoria dell'attaccamento offra una chiave interpretativa della relazione tra trauma e psicopatologia, dimostrando l'importanza delle relazioni di attaccamento, come fattore di rischio o protezione nella costruzione del sé laddove il maltrattamento, nelle sue diverse espressioni, rappresenta un trauma evolutivo (Caviglia, Perrella, La Marra, \& Bisogno, 2007).

Un altro lavoro a cui ha partecipato un membro del gruppo, ha studiato la dissociazione e il trauma in una paziente di 24 anni, sottoposta a psicoterapia a orientamento analitico, a cui sono stati somministrati l'AAI, l'OPD (Diagnosi Psicodinamica Operazionalizzata) e la SWAP-200. All'inizio della psicoterapia, la narrazione della perdita della nonna materna mostrava aspetti dissociativi nell'uso del 
linguaggio, mentre il lavoro di psicoterapia ha aumentato il processo referenziale, portando alla riconnessione dei diversi sistemi di codifica sub-simbolici e simbolici (De Coro, Caviglia, Giannini, Iberni, \& Mariani, 2008).

Negli ultimi anni, la riflessione del Gruppo sul trauma si è orientata verso la Psicologia delle emergenze (Caviglia \& Nardiello, 2009; Nardiello, La Marra, Fedeli, \& Del Villano, 2009; Nardiello, Perrella, La Marra, Sapuppo, Bisogno, \& Caviglia, 2009; Nardiello, Pinto, \& Sapuppo, 2009), come campo clinico dentro il quale andare a studiare i rapporti tra eventi traumatici e disturbi psichici. Il Gruppo di lavoro specifico sulla ricerca in Psicologia delle Emergenze è impegnato nell'elaborazione di modelli e procedure di intervento nell'ambito della prevenzione primaria e secondaria dei disturbi post-traumatici, anche grazie alla collaborazione diretta con l'Ordine degli Psicologi della Regione Campania che, con il Corpo Militare della Croce Rossa Italiana, organizza e espleta seminari e corsi di formazione, diretti a Psicologi e a personale di soccorso coinvolto nella gestione delle emergenze. Tale attività, fornendo conoscenze teoriche e operative in contesti di emergenza, rappresenta il primario elemento preventivo di problematiche derivanti dall'incontro con il trauma. Il personale formato è seguito prima e dopo le operazioni in emergenza per il monitoraggio delle loro dinamiche psichiche legate all'evento e per la raccolta di informazioni e dati per la ricerca. La finalità è quella di proporre, alle organizzazioni che si interessano di emergenza, un più efficace piano d'intervento per l'ambito del supporto clinico e psicologico di vittime, soccorritori e tecnici impegnati nel contesto delle catastrofi.

\section{5) Ambito psicodiagnostico}

Il Gruppo di ricerca si occupa dei reattivi psicodiagnostici maggiormente diffusi (Rorschach, DAP, WAIS, MMPI), che permettono allo Psicologo di orientarsi verso un determinato inquadramento 
diagnostico. Il tentativo è quello di verificare le convergenze di indici di strumenti diversi, al fine di apportare un contributo nella comprensione e valutazione di soggetti normali e/o patologici, anche nelle procedure di assessment richieste a livello istituzionale (ASL, Tribunali, Dipartimenti di medicina legale, etc.). Il clinico ha a disposizione una serie di strumenti per la diagnosi, che vanno dalle scale di valutazione in psichiatria ai test proiettivi. Il loro utilizzo è subordinato all'analisi della domanda, al colloquio clinico, alla situazione diagnostica, al contesto, alla committenza e al quesito posto dall'utente a livello collusivo. Il tentativo del Gruppo (Caviglia, 2005; Caviglia \& Del Castello, 2003; Caviglia \& Pinto, 2009; La Marra, 2006) è stato quello di coniugare intervento clinico e riflessione teorica, mostrando praticamente l'applicazione, in casi clinici reali, di approcci valutativi di analisi della domanda e testologici. Inoltre, per quanto concerne la depressione (Perrella, 2006), il Gruppo di ricerca ha approfondito lo studio teorico e l'utilizzo di diversi test per valutare tale patologia nell'infanzia e nell'adolescenza. Sono stati trattati il Test dell'ansia e della depressione nell'infanzia e nell'adolescenza (TAD; Newcomer, Barenbaum, \& Bryant, 1995), il Children's Depression Inventory (CDI; Kovacs, 1988) e la Children's Depression Scale (CDS; Lang \& Tisherm, 1978). Tra i reattivi utilizzati per effettuare diagnosi di depressione nei soggetti adulti sono stati utilizzati il Beck Depression Inventory (BDI; Beck, Ward, Mendelson, Mock, \& Erbaugh, 1961), la Hamilton Depression Rating Scale (HDRS o HAM-D; Hamilton, 1960), la SelfRating Depression Scale (SDS; Zung, 1965). In altri lavori, sono stati utilizzati strumenti specifici per la diagnosi psicologica nell'alcolismo (Aurilio, Palmisciano, \& Caviglia, 2004), quali il MALT, il MAST, il CAGE (Ewing, 1984) e si è affrontata la riflessioni sul problema della "doppia diagnosi" (Caviglia, La Marra, \& Bonifacio, 2006). 
Ricerca in Psicoterapia / Research in Psychotherapy 2010; 2 (13): 32-52 http://www.researchinpsychotherapy.net

\section{Bibliografia}

Andreassi, S., Cascioli, A., Caviglia G., De Coro, A., Lingiardi, V., Ortu, F., Pazzagli, C., Piscicelli, S., Williams, R., \& Dazzi, N. (2000, Settembre). Ricerca concettuale e ricerca empirica nella psicoterapia psicodinamica: una metodologia per lo studio del processo. Relazione presentata al II Congresso Nazionale della Sezione di Psicologia Clinica della A.I.P., Alghero.

Attili, G. (2001). Ansia da Separazione e misura dell'Attaccamento normale e patologico. Milano: Edizioni Unicopli.

Ausilio, R., Palmisciano, L., \& Caviglia, G. (2004). La diagnosi psicologica nell'alcolismo: problemi e soluzioni mediante l'uso di test. Antologia Medica Italiana, XXIV, 157-167.

Baiocco, R., Giannini, A.M., \& Laghi, F. (2005). Scala Alessitimica Romana. Trento: Erikson.

Beck, A.T., Ward, C.H., Mendelson, M., Mock, J., \& Erbaugh, J. (1961). An Inventory for Measuring Depression. Archives of General Psychiatry, 4, 56171.

Bernstein, E.M., \& Putnam, F.W. (1986). Development, reliability and validity of a dissociation scale. The Journal of Nervous and Mental Disease, 174(12), 727-735.

Bowlby, J. (1973). Attaccamento e perdita, Vol. 2: La separazione dalla madre. Tr. it. Boringhieri, Torino 1975.

Brisch, K.H. (2005). I Disturbi dell'Attaccamento. Dalla teoria alla terapia. Tr. it. a cura di G. Caviglia, Fioriti, Roma 2007.

Bucci, W. (1997). Psicoanalisi e scienza cognitiva. Tr. it. Fioriti, Roma 1999.

Carcione, A., Falcone, M., Magnolfi, G., \& Manaresi, F. (1997). La funzione metacognitiva in psicoterapia: scala di valutazione della metacognizione (S.Va.M.). Psicoterapia, 9, 91-107.

Cassibba, R., \& Caviglia, G. (2000). La relazione educatrice-bambino all'asilo nido: variabili rilevanti per il benessere del bambino. Psichiatria dell'Infanzia e dell'Adolescenza, 67(1), 85-92.

Caviglia, G. (1998). Il concetto di trauma nella letteratura psicodinamica. Psichiatria dell'Infanzia e dell'Adolescenza, 65(3), 355-366.

Caviglia, G. (2001). L'Attività Referenziale (R.A.) di Wilma Bucci: fra terapia, ricerca psicodinamica e teoria psicoanalitica. Psichiatria dell'Infanzia $e$ dell'Adolescenza, 68(3), 319-328.

Caviglia, G. (2003). Attaccamento e psicopatologia. Roma: Carocci.

Caviglia, G. (2005). Teoria della mente, attaccamento disorganizzato, psicopatologia. Roma: Carocci.

Caviglia, G. (Ed.). (2005). Il lavoro diagnostico dello psicologo clinico: teoria, strumenti, competenze. Caserta: Edizioni Melagrana Onlus.

Caviglia, G. (2009). Disturbi del Comportamento Alimentare, fenomeni dissociativi ed esperienze traumatiche. A Scuola, VIII(11), 12-13.

Caviglia, G., \& Bisogno, S. (2008). Qualità dell'Attaccamento e sviluppo della "Teoria della mente". Psichiatria dell'infanzia e dell'adolescenza, 75(1), 87-98.

Caviglia, G., \& Bisogno, S. (2009, June). Intergenerational transmission of the trauma of Holocaust: a study conducted by the Adult Attachment Interview in an Italian sample. Paper presented at $4^{\text {th }}$ International Conference of the International Association for Relational Psychoanalysis and Psychotherapy (IARPP), "The Shadow of Memory: Relational Perspectives on Remembering and Forgetting", Tel Aviv, Israel. 
Ricerca in Psicoterapia / Research in Psychotherapy 2010; 2 (13): 32-52 http://www.researchinpsychotherapy.net

Caviglia, G., Bisogno, S., Perrella, R., \& La Marra, M. (2006, Settembre). Stati dissociativi nei Disturbi del Comportamento Alimentare. Relazione presentata al VI Congresso Nazionale della SPR, Reggio Calabria.

Caviglia, G., Cassibba, R., \& Coppola, G. (2000, Settembre). L'uso dell'Attachment Story Completion Task nello studio delle relazioni tra I.W.M., abilità linguistiche in età prescolare e Attività Referenziale. Relazione presentata al II Congresso Nazionale della Sezione di Psicologia Clinica della A.I.P., Alghero.

Caviglia, G., \& Cecere, F. (2007). I disturbi del comportamento alimentare. L'approccio multidisciplinare per un intervento efficace. Roma: Carocci.

Caviglia, G., \& De Coro, A. (2000). Ricerca empirica e cambiamento terapeutico: lo studio dell'attività referenziale tra psicoanalisi e psicologia cognitiva. In A. De Coro \& G. Caviglia, Manuale per il calcolo dell'Attività Referenziale. Roma: Kappa.

Caviglia, G., \& Del Castello, E. (Eds.). (2003). La diagnosi in Psicologia Clinica. Milano: Franco Angeli.

Caviglia, G., Del Castello, E., \& Curcio, L. (2001, Settembre). Attaccamento ed Agorafobia: uno studio clinico sulle coppie. Relazione presentata al III Congresso Nazionale della Sezione di Psicologia Clinica della A.I.P., Palermo.

Caviglia, G., Del Castello, E., \& Fiocco, B. (2001, Ottobre). Analisi dello stato della mente rispetto all'attaccamento, indagato con l'Adult Attachment Interview, in donne con Agorafobia e nei loro mariti: uno studio pilota. Relazione presentata al III Convegno Nazionale della S.P.R. Italia, Palermo.

Caviglia, G., Del Castello, E., \& Fiocco, B. (2002). L'Attaccamento in donne agorafobiche e nei loro partner: una ricerca empirica. Ricerca in Psicoterapia, 5(3), 187-206.

Caviglia, G., \& Del Villano, N. (2010). Attività Referenziale, Dissociazione e Psicopatologia. Psichiatria e Psicoterapia, XXIX(3), 139-155.

Caviglia, G., Del Villano, N., \& Sapuppo, W. (2010). Raccontare la propria vita: tre metodi a confronto fra Psicologia, Psicoterapia e Pedagogia. Nuovi Orizzonti, Semestrale di Psicologia, Medicina e Pedagogia, II(3), 4-9.

Caviglia, G., \& Fiocco, B. (2003, Novembre). La trasmissione intergenerazionale del trauma della Shoà: uno studio condotto con l'Adult Attachment Interview. Relazione presentata al IV Congresso Nazionale della S.P.R. Italia, Milano.

Caviglia, G., Fiocco, B., \& Dazzi, N. (2004). La trasmissione intergenerazionale del trauma della Shoà: uno studio condotto con l'Adult Attachment Interview. Ricerca in Psicoterapia, 7(1), 67-83.

Caviglia, G., Fiocco, B., \& Fullone, A. (2003, Settembre). La trasmissione intergenerazionale del trauma della Shoà indagata con l'Adult Attachment Interview, l'analisi del contenuto e l'analisi delle corrispondenze lessicali. Relazione presentata al V Congresso Nazionale della Sezione di Psicologia Clinica della A.I.P., Bari.

Caviglia, G., Fiocco, B., Solla, A., \& Dazzi, N. (2004, Giugno). Intergenerational transmission of Shoà trauma: A research carried out with the Adult Attachment Interview. Paper presented at 35th Annual Meeting della Society for Psychotherapy Research, Roma.

Caviglia, G., Gangi, S., \& Fiocco, B. (2002, Settembre). L'Adult Attachment Interview nello studio della trasmissione intergenerazionale del trauma della Shoà. Relazione presentata al IV Congresso Nazionale della Sezione di Psicologia Clinica della A.I.P., Bellaria-Rimini. 
Ricerca in Psicoterapia / Research in Psychotherapy 2010; 2 (13): 32-52 http://www.researchinpsychotherapy.net

Caviglia, G., Iuliano, C., \& Perrella, R., (2005). Il disturbo borderline di personalità. Roma: Carocci.

Caviglia, G., La Marra, M., \& Bonifacio, M. (2006). Alcolismo e comorbilità psichiatrica: la doppia diagnosi per un intervento mirato. In R. Malinconico (Ed.). Le droghe: uso, disuso, abuso. Caserta: Edizioni Melagrana Onlus.

Caviglia, G., La Marra, M., Sapuppo, W., \& Perrella, R. (2010). Automated Working Memory Assessment e apprendimento nei bambini: una ricerca empirica. Psichiatria dell'Infanzia e dell'Adolescenza, 77, 38-47.

Caviglia, G., \& Nardiello, D. (Eds.). (2009). Le dinamiche psicologiche nelle emergenze. Napoli: Idelson Gnocchi.

Caviglia, G., Perrella, R., Bisogno, S., \& La Marra, M. (2006, Settembre). Dissociazione e alessitimia in alcuni dei disturbi delle condotte alimentari. Congresso Nazionale di Psicologia Clinica dell'AIP, Rovereto.

Caviglia, G., Perrella, R., La Marra, M., \& Bisogno, S. (2007). Esiti psicopatologici del trauma e dell'abuso: ricerche empiriche e modelli eziopatogenetici. Psichiatria dell'infanzia e dell'adolescenza, 74(1), 183-193.

Caviglia, G., Perrella, R., La Marra, M., \& Giannini, F. (2006). Disturbi alimentari in adolescenza, Dissociazione e Alessitimia. Psichiatria dell'infanzia e dell'adolescenza, 73(3-4), 615-625.

Caviglia, G., \& Pili, M. (2001). Attaccamento sicuro e sviluppo delle competenze socio-emotive in bambini in età scolare. Psichiatria dell'Infanzia e dell'Adolescenza, 68(5-6), 571-582.

Caviglia, G., \& Pinto, P. (2009). Il lifelong learning visto da una prospettiva psicoanalitica. In B. Schettini, (Ed.). Governare il lifelong learning. Prospettive di educazione degli adulti. Napoli: Edizioni Scientifiche Italiane.

Caviglia, G., Scafuto, F., Perrella, R., \& Semerari, A. (2006). Processi metacognitivi e disturbo borderline di personalità: studio di un caso singolo attraverso la S.Va.M. Cognitivismo clinico, 3(1), 21-29.

Caviglia, G., Scafuto, F., Solla, A., \& Semerari, A. (2003, Settembre). Processi metacognitivi e disturbo borderline di personalità: studio di un caso singolo attraverso la scala S.Va.M. Relazione presentata al V Congresso Nazionale della Sezione di Psicologia Clinica della A.I.P., Bari.

Caviglia, G., Solla, A., \& Dazzi, N. (2003). Attaccamento e problemi comportamentali ed emozionali: uno studio su bambini di 6 anni. Infanzia e adolescenza, 2, 68-77.

Caviglia, G., Solla, A., Scafuto, F., \& Semerari, A. (2003, Novembre). Studio del processo di cambiamento in psicoterapia di un paziente con disturbo borderline di personalità, attraverso la scala S.Va.M. Relazione presentata al IV Congresso Nazionale della S.P.R.-Italia, Milano.

Cianchetti, C., \& Sannio Fancello, G. (2007). TVL - Test di Valutazione del Linguaggio. Gardolo (TN): Erickson.

De Coro, A., \& Caviglia, G. (Eds.). (2000). La valutazione dell'Attività Referenziale. Roma: Kappa.

De Coro, A., Caviglia, G., Giannini, F., Iberni, E., \& Mariani, R. (2008). Dissociazione e trauma nella teoria del codice multiplo. In V. Caretti \& G. Craparo, Trauma e psicopatologia. Un approccio evolutivo-relazionale. Roma: Astrolabio.

De Coro, A., Ortu, F., Caviglia, G., Andreassi, S., Pazzagli, C., Mariani, R., Visconti, E., Bonfanti, A., Bucci, W., \& Maskit, B. (2004, Giugno). Referential Activity and verbal exchanges in psychotherapy: A first clinical application of 
Ricerca in Psicoterapia / Research in Psychotherapy 2010; 2 (13): 32-52 http://www.researchinpsychotherapy.net

the Italian WRAD version to the study of a single case. Paper presented at the 35th Annual Meeting della S.P.R. Roma.

Del Villano, N., Bisogno, S. (2009, Settembre). Narrazione Autobiografica e Adult Attachment Interview: due metodi a confronto. Relazione presentata al XI Congresso Nazionale della Sessione di Psicologia Clinica e Dinamica dell'AIP, Chieti. Atti del convegno, pp. 277-278.

Del Villano, N., Sapuppo, W., Cecere, C., La Marra, M., Perrella, R., Marciano, R., Intoccia, T., \& Caviglia, G. (2010, Settembre). Working Memory, Apprendimento e Stile di attaccamento: una ricerca empirica in progress nella pratica educativa. Relazione presentata al XII Congresso Nazionale della Sezione di Psicologia Clinica e Dinamica dell'AIP, Torino. Abstract Book, p. 248.

Ewing, A.J., (1984), Detecting alcoholism: the CAGE questionnaire. Journal of Medical Association, 252, 1905-1907.

Garner D.M. (1991): EDI-2. Eating Disorder Inventory-2. Professional Manual, Odessa FL: Psychological Assessment Resource Inc.

George, C., Kaplan, N., \& Main, M. (1985). Adult Attachment Interview. Manoscritto non pubblicato. Berkeley: University of California.

Hamilton, M. (1960). A rating scale for depression. J. Neurol. Neurosurg. Psychiat, 23(56), 50-55.

Harms, T., Cryer, D., Clifford, R.M., (1990). Tr. it. a cura di Ferrari M., Livraghi P., Svani. Scala per la valutazione dell'asilo nido. Franco Angeli, Milano 1992.

Hutton, J.B., \& Roberts, T.G. (1986). Test di valutazione dei problemi comportamentali ed emozionali. Tr. it. Centro Studi Erickson, Trento 1989.

Kovacs, M. (1988). Children's Depression Inventory (CDI). In M. Camuffo, R. Cerutti, L. Lucarelli, \& R. Mayer (Eds.), Children's Depression Inventory. Questionario di autovalutazione. Firenze: Giunti O.S.

La Marra, M. (2006). Gli strumenti per la diagnosi. In R. Perrella (Ed.), La depressione: storia, teoria, clinica. Roma: Carocci Editore.

La Marra, M., Bisogno, S., Perrella, R., \& Caviglia, G. (2006, Ottobre). Presenza dei disturbi alimentari in un gruppo di operatori dello spettacolo. Relazione presentata al VI Congresso Nazionale della Società per la Ricerca in Psicoterapia, Reggio Calabria.

La Marra, M., Perrella, R., Bisogno, S., \& Caviglia, G. (2006, Settembre). Valutazione dei disturbi alimentari e Operatori dello Spettacolo: una ricerca empirica. Relazione presentata al Congresso Nazionale di Psicologia Clinica dell'AIP, Rovereto.

La Marra, M., Perrella, R., Marciano, R., Cecere, C., Intoccia, T., Ciccarelli, M.A., Del Villano, N., Bisogno, S., \& Caviglia, G. (2009, Settembre). Working Memory, Apprendimento e Modelli Operativi Interni nei bambini: una ricerca empirica in progress. Relazione presentata al XI Congresso Nazionale della Sessione di Psicologia Clinica e Dinamica dell'AIP, Chieti. Atti del convegno, pp. 282-283.

La Marra, M., Piombino, L., Messina, G., Perrella, R., Chieffi, S., Mangoni di Santo Stefano, C., \& Caviglia, G. (2006, Settembre). Dissociation and alexithymia in eating disorders. Relazione presentata al Congresso Nazionale della Società Italiana di Fisiologia, Ravenna.

La Marra, M., Sapuppo, W., \& Caviglia, G. (2008). La psicopatia e il Disturbo Antisociale di Personalità: aspetti storici, teorici e clinici. Relazione presentata al I Convegno Nazionale di Psicologia Giuridica, Università degli Studi di Bari. 
Ricerca in Psicoterapia / Research in Psychotherapy 2010; 2 (13): 32-52 http://www.researchinpsychotherapy.net

La Marra, M., Sapuppo, W., \& Caviglia, G. (2009a). Caratteristiche temperamentali e caratteriali nell'obesità in adolescenza. Psichiatria dell'infanzia e dell'adolescenza, 76(1), 225-236.

La Marra, M., Sapuppo, W., \& Caviglia, G. (2009b). Dissociazione e alessitimia in un campione di pazienti con Disturbo del Comportamento Alimentare. Ricerca in psicoterapia/Research in psychotherapy: Psychopathology, Process and Outcome, 12(1/2), 6-20.

La Marra, M., Sapuppo, W., \& Caviglia, G. (2010, Settembre). Caratteristiche temperamentali e caratteriali dell'obesità in adolescenza. Relazione presentata al XII Congresso Nazionale della Sezione di Psicologia Clinica e Dinamica dell'AIP, Torino.

La Marra, M., Sapuppo, W., Chieffi, S., \& Caviglia, G. (2010, Settembre). Caratteristiche temperamentali e caratteriali dell'obesità in adolescenza. Relazione presentata al XII Congresso Nazionale della Sezione di Psicologia Clinica e Dinamica dell'AIP, Torino.

La Marra, M., Sapuppo, W., Messina, G., Mangoni Di S. Stefano, C., Chieffi, S., \& Caviglia, G. (2009). Tratti temperamentali e caratteriali in un campione di adolescenti campani. Relazione presentata alle Giornate Scientifiche di Ateneo 2009 Sessione VIS-2: Scienze della Vita e della Salute, Seconda Università degli Studi di Napoli.

Lang, M., \& Tisher, M. (1983). Children's Depression Scale (CDS). In S. Savellini-Gori \& F. Abbele-Morino (Eds.), (1984), Scala di valutazione di Depressione. Firenze: Giunti O.S.

Liotti, G., Guidano, V. (1976). Behavioural analysis of marital interaction in agoraphobic male patient. Behaviour research and therapy, 14, 161-162.

Liverta Sempio, O., Marchetti, A., \& Leccio, F. (2001). Il SAT-famiglia e il SATscuola. Strumenti di misura dell'ansia da separazione da genitori ed insegnanti. Milano: I.S.U. Università Cattolica.

Lyons-Ruth, K. \& Atwood, G. (1997). Identification with a Hostile or Helpless attachment figure: Additional correlate of infant disorganization in Adult Attachment Interview. In L. Atkinson \& J. Zucker (Eds.), Attachment and Psychopathology, vol. 2. Cambridge: Cambridge University Press.

Moses, R. (Ed.). (1993). The persistent shadows of the Holocaust: The meaning to those not directly effected. Madison, CT: International University Press.

Nardiello, D., La Marra, M., Fedeli, F., \& Del Villano, N. (2009). Comunicazioni e Tecniche, specifiche e immediate, nella Psicologia dell'Emergenza. In G. Caviglia \& D. Nardiello (Eds.), Le Dinamiche Psicologiche nelle Emergenze. Napoli: Idelson-Gnocchi.

Nardiello, D., Perrella, R., La Marra, M., Sapuppo, W., Bisogno, S., \& Caviglia, G. (2009). Le dinamiche psicologiche negli scenari delle catastrofi. Relazione presentata al XI Convegno Nazionale Ufficiali Medici Croce Rossa Italiana, IX simposio personale sanitario C.R.I.

Nardiello, D., Pinto, P., \& Sapuppo, W. (2009). Il soccorritore e la vittima: le figure dell'emergenza. In G. Caviglia \& D. Nardiello (Eds.), Le dinamiche psicologiche nelle emergenze. Napoli: Idelson Gnocchi.

Newcomer, P., Barembaum, E., \& Bryant, B. (1995). Depression and Anxiety in Youth Scale. In D. Ianes (Ed.). Test dell'Ansia e della Depressione nell'infanzia e adolescenza (TAD). Trento: Erikson.

Pederson, D.R., \& Moran, G. (1995). Appendix B. Maternal Behavior Q-set. In E. Waters, B.E. Vaughn, G. Poseda, \& K. Kondo-Ikemura (Eds.), Caregiving, cultural, and cognitive perspectives on secure-base behavior and working 
Ricerca in Psicoterapia / Research in Psychotherapy 2010; 2 (13): 32-52 http://www.researchinpsychotherapy.net

models: New Growing Points of Attachment Theory and Research. Monographs of the Society for Research in Child Development, 60, 2-3, Serial No. 244.

Perrella, R. (2006). La Depressione: storia, teoria, clinica. Roma: Carocci Editore.

Perrella, R., D’Amore, G., La Marra, M., \& Caviglia, G. (2005, Settembre). L'intervento psicologico con il paziente borderline: il processo di cambiamento in un caso clinico. Relazione presentata al Congresso Nazionale della Sezione di Psicologia Clinica dell'AIP, Cagliari.

Tagini, A., Pazzagli, C., Caviglia, G., \& Dazzi, N. (2007, Settembre). Stato della mente rispetto all'attaccamento, attività referenziale e memoria autobiografica: uno studio pilota su soggetti 'Non Classificabili'. Relazione presentata al IX Congresso Nazionale della Sezione di Psicologia Clinica e Dinamica dell'AIP, Perugia.

Tagini, A., Pazzagli, C., Caviglia, G., De Coro, A., \& Dazzi, N. (2008). States of mind with respect to attachment and Referential Activity: A pilot study on traumatic autobiographical memories. Paper presented at the 39th SPR International Annual Meeting, Barcellona.

Tagini, A., Pazzagli, C., Caviglia, G., Pravato, D., De Coro, A., \& Dazzi, N. (2009). Traumi infantili e attaccamento adulto: uno studio pilota con l'Adult Attachment Interview e il DAAP. Quaderni CPD, 6, numero speciale monotematico su: "Il Processo Referenziale: studi clinici e ricerca empirica", 39-56.

van Ijzendoorn, M.H., \& Bakermans-Kranenburg, M.J. (1996). Attachment representation in mothers, fathers, adolescents and clinical groups: a meta-analytic search for normative data. Journal of Consulting and Clinical Psychology, 64(1), 8-21.

Waters, E., \& Deane, K.E. (1985). Defining and assessing individual differences in attachment relationships: Q-methodology and the organization of behavior in infancy and early childhood. In I. Bretherton \& E. Waters (Eds.), Growing points of attachment theory and research. Monographs of the Society for Research in Child Development, 50, 1-2, 209.

Zung, W.W.K. (1965). A Self-rating Depression Scale. Archives of General Psychiatry, 12, 63-70.

\begin{abstract}
The purpose of this article is to illustrate the current interests of our italian Research Group link with the course of "Dynamic Psychology" (basic) of the Faculty of Psychology at $2^{\text {nd }}$ University of Neaples and the works published in recent years resulting from these investigations. The works are placed in areas related to Attachment Theory, psychological assessment, study of psychopathology related to Eating Disorders and psychotherapy research "single case".
\end{abstract}

\title{
Keywords
}

Eating disorders, attachment theory, psychotherapy research, psychopathology 